\title{
Modeling of capacitance transients of thin-film solar cells: a valuable tool to gain information on perturbing layers or interfaces
}

\author{
Johan Lauwaert, ${ }^{1, *}$ Jeroen Lauwaert, ${ }^{2}$ Lisanne Van \\ Puyvelde, ${ }^{1}$ Joris W. Thybaut, ${ }^{2}$ and Henk Vrielinck ${ }^{1}$ \\ ${ }^{1}$ Department of Solid State Sciences, Ghent University, \\ Krijgslaan 281-S1, 9000 Gent, Belgium \\ ${ }^{2}$ Laboratory for Chemical Technology (LCT), \\ Ghent University, Krijgslaan 281-S5, 9000 Gent, Belgium
}

(Dated: January 7, 2014)

\begin{abstract}
Thin-film electronic and photovoltaic devices often comprise, in addition to the anticipated p-n junctions, additional non-ideal ohmic contacts between layers. This may give rise to additional signals in capacitance spectroscopy techniques that are not directly related to defects in the structure. In this paper we present a fitting algorithm for transient signals arising from such an additional junction. The fitting results are in excellent agreement with the diode characteristics extracted from static measurements on individual components. Finally the algorithm is applied for determining the barriers associated with anomalous signals reported for selected $\mathrm{CuIn}_{1-\mathrm{x}} \mathrm{Ga}_{\mathrm{x}} \mathrm{Se}_{2}$ and CdTe solar cells.
\end{abstract}

PACS numbers: $71.55 . \mathrm{Cn}$

*Electronic address: Johan.Lauwaert@UGent. be 
Thin-film electronic devices are multiple-layer structures. Their production implies several deposition and processing steps, each of which may influence all layers already present. Electric characterization with capacitance-voltage profiling, admittance spectroscopy (AS) and Deep Level Transient Spectroscopy (DLTS)[1, 2] is often applied to assess the quality of such devices and to understand the impact of defects on their properties and performance. Progress in the development of components depends on understanding the influence of each layer on the device's electric properties. Although studying isolated layers or simplified devices is valuable, its relevance for the complete device is not guaranteed. Moreover, even single or double-layer structures need making of electric contacts, which may influence the observed results. Hence, characterization of finished products provides the most relevant information and is preferred by the manufacturers. However, in complete devices the assignment of observed effects to particular layers and even the interpretation of certain features, is often quite difficult. DLTS is traditionally applied to study carrier trapping by electrically active defects in semiconductors. $[1,2]$ Spectra recorded on thin-film devices are usually interpreted in terms of defects in particular layers.

Recently, in the context of interpretation of the capacitance spectroscopy signals observed for $\mathrm{CuIn}_{1-\mathrm{x}} \mathrm{Ga}_{\mathrm{x}} \mathrm{Se}_{2}$ (CIGS) solar cells, we have studied the DLTS characteristics of a nonOhmic contact in layered structures, acting as a p-n junction polarized oppositely to the main junction. We have found that such a contact may give rise to a strong DLTS signal that can readily be distinguished from that of defects by comparing DLTS results after regular and inverted electric pulses.[3, 4] In case of a regular pulse, the transient is recorded in depletion at $V_{r}$ after applying a pulse from $V_{r}$ to $V_{p}$ for which $V_{r}<V_{p}<0$, and for an inverted pulse $V_{p}<V_{r}<0$. The resulting conventional (after regular pulse) and complementary signals (after inverted pulse) obviously have different signs. Although in principle the conventional signal of a non-ideal contact can have either sign, it is very often negative, this means, decreasing in time. It is interesting to note that this excludes both defects in certain layers or defect states at interfaces between layers. The amplitude of the transients for these defects is of course strongly dependent on their concentration and localization. Nevertheless, for majority carriers a transient is rising for emission and falling for capture. For the DLTS signal of the emission and capture from a distribution of interface states, e.g. in a metal-oxide-semiconductor capacitor in depletion or accumulation the signals show the same signs as observed for bulk defects.[5-7] A similar defect-like behavior is expected for 
trapping of majority carriers in high-k dielectrics. [8-10]

The DLTS signals of non-ideal contacts may be very intense and hamper the actually intended detection of carrier trap levels.[11] Nonetheless, they may also provide very valuable information on the thin-film device if they allow to extract the electric (diode) characteristics of the non-ideal contact, e.g. its barrier height and saturation current. Such information may help to identify the corresponding contact layer, provide input parameters for assessing its (detrimental or beneficiary) effect on the device properties through simulations, and through all this, direct the device optimization.

In this paper we demonstrate that via fitting the DLTS spectra with regular and inverted pulses of various heights, these parameters can be extracted. The described fitting algorithm is validated on a model device consisting of a series connection of two oppositely polarized diodes (pn BA102 and 3 Cr-Si Schottky diodes 1N5819 in parallel), whose characteristics are determined by static measurements on the individual diodes. The use of this fitting tool is further illustrated by determining the barrier height of the non-Ohmic contact that gives rise to the N1 signal for two CIGS cells [12-14] with different buffer layers and for a $\mathrm{CdTe} / \mathrm{CdS}[15,16]$ solar cell, whose characteristics have been discussed before. Conventional $\left(V_{r}-V_{p}<0\right)$ and complementary $\left(V_{r}-V_{p}>0\right)$ DLTS spectra[3] were recorded using a PhysTech DLTS spectrometer.

The model circuit we use to calculate the properties of the capacitance transients observed in DLTS is a normal diode perturbed with another diode as barrier. The electric properties of both components are modeled including their current and capacitance characteristics. The total current through both components $(I)$ as a function of voltage $(V)$ over the respective component is given by:

$$
I=I(V)+C(V) \frac{d V}{d t}
$$

with $I(V)$ the quiescent current and $C(V)$ the capacitance which are dependent on the potential drop over the component. For the main junction (M) the I-V characteristics of a reverse polarized diode are calculated as thermionic emission over a barrier $\Delta E_{M}[17]$ :

$$
I_{M}=A_{M} T^{2} \exp \left(-\frac{\Delta E_{M}}{k_{B} T}\right)\left(\exp \left(\frac{-q V_{M}}{k_{B} T}\right)-1\right)
$$

while the capacitance is that of a depletion layer having a uniform doping and a straight 
Mott-Schottky behavior:

$$
C_{M}=\frac{C_{M 0}}{\sqrt{1+\frac{V_{M}}{V_{M 0}}}}
$$

The properties of the additional barrier (B) are also modeled using thermionic emission. This additional barrier is forward biased, because the main diode is continuously biased in reverse (i.e. $V_{r}<0$ and $\left.V_{p}<0\right)$ during the DLTS experiment. Hence,

$$
I_{B}=A_{B} T^{2} \exp \left(-\frac{\Delta E_{B}}{k_{B} T}\right)\left(1-\exp \left(\frac{q V_{B}}{k_{B} T}\right)\right)
$$

Because the potential drop over this barrier is small, only a linear dependence on forward bias $V_{B}$ of the capacitance is taken into account:

$$
C_{B}=C_{0 B}+\alpha_{B} V_{B}
$$

Besides these two components a series resistance $R$ is included to avoid discontinuities in the circuit response. The accuracy of the resulting fit parameters will depend strongly on the validity of the model. Although this model based on two barriers obeying thermionic emission is an evident choice for the model circuit, the $\mathrm{I}-\mathrm{V}$ and $\mathrm{C}-\mathrm{V}$ characteristics of an additional barrier in thin-film devices are in general difficult to predict.

Based on this model the instantaneous capacitance at an observation frequency $\nu=1 \mathrm{MHz}$ can be calculated as a function of the potential drops $V_{B}$ and $V_{M}$ and the capacitances $C_{B}$ and $C_{M}$

$$
C=\frac{C_{B} V_{B}^{2}+C_{M} V_{M}^{2}+4 \pi^{2} \nu^{2} V_{B}^{2} V_{M}^{2} C_{B} C_{M}\left(C_{B}+C_{M}\right)}{\left(V_{B}+V_{M}\right)^{2}+4 \pi^{2} \nu^{2} V_{B}^{2} V_{M}^{2}\left(C_{M}+C_{B}\right)^{2}}
$$

The quiescent bias over the whole structure or circuit equals $V_{r}$ during observation and $V_{p}$ during the pulse:

$$
V_{r / p}=V_{B}+V_{M}+R I
$$

The time evolution of $V_{M}, V_{B}, C_{M}, C_{B}$ and the current $I$ is calculated with a finite differences method for given parameters of $\mathrm{M}\left(A_{M}, E_{M}, C_{0 M}, V_{M 0}\right), \mathrm{B}\left(A_{B}, E_{B}, C_{0 B}, \alpha_{B}\right)$, measurement parameters $V_{r}, V_{p}, t_{0}, T_{w}, t_{p}$ and the temperature $T$. In this way we implemented the solution with 8 unknown parameters to be determined by fitting with 6 experimental (known) input parameters in a non-linear fitting algorithm.[18] Although the whole transient contains the necessary information to unravel the properties of the back contact, taking into account all points would make the calculations very time consuming. Therefore the optimization 


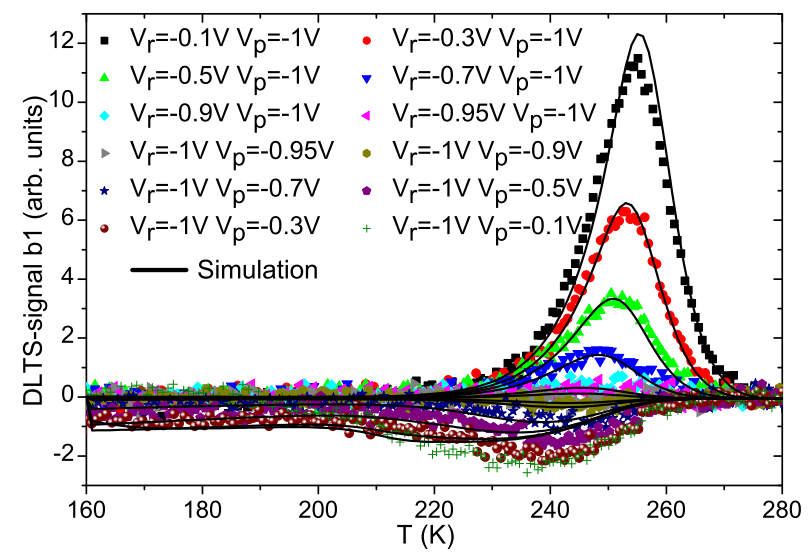

FIG. 1: DLTS spectrum recorded for different biases on a model circuit

algorithm uses only the first four Fourier components of the capacitance transient $b_{1}, a_{1}, b_{2}$ and $a_{2}[19]$ :

$$
a_{i}+j b_{i}=\frac{1}{T_{w}} \int_{t_{0}}^{T_{w}+t_{0}} C(t) \exp \left(j \frac{2 i \pi}{T_{w}} t\right) d t
$$

As the transients are not necessarily single exponential and the amplitude of the observed transient can depend on temperature, these components provide independent information. The number of data points (experiments) were reduced to limit the computer time needed for the algorithm to simulate the DLTS spectra for a given trial set of parameters. For the model circuit, e.g., only the interval $180,280 K$ with step $\Delta T=10 K$ was used, while for the biases the experiments with the lowest difference in reverse bias and pulse $\left(\left\|V_{r}-V_{p}\right\|=\Delta V\right)$ were omitted. Extra weighing of the data was not included in the algorithm, which means that the transients with the largest amplitude have the strongest effect on the results, but these signals also exhibit the highest signal to noise ratio. For the model circuit this results in 66 experiments with 6 input parameters $V_{r}, V_{p}, T, t_{0}, T_{w}, t_{p}$ and 4 output parameters $b_{1}, a_{1}, b_{2}, a_{2}$ (i.e. $11 \times 6 \times 4=264$ data points). A similar reduction in data was used for the solar cells. Although the program uses complete calculations of the transients, it is instructive to make further simplifications. All the signals observed are in a regime for which the time constant of the main junction $\tau_{M}$ is larger than the time constant of the barrier $\tau_{B}\left(\tau_{M}>\tau_{B}\right)$. This can e.g. be seen in the spectra for the model circuit shown in Fig. 1. The circuit response 
$\left(\tau_{\text {circuit }}\right)$ is much faster than the time constants of the barrier $\tau_{B}$ and the main junction $\tau_{M}$ :

$$
\tau_{\text {circuit }}=\frac{C_{M} C_{B} R}{C_{M}+C_{B}}<<\tau_{B}<\tau_{M}
$$

In such a situation the observed time constant can be approximated by [3]:

$$
\tau \approx \frac{V_{M} V_{B}}{I\left(V_{M}+V_{B}\right)}\left(C_{M}+C_{B}\right) \approx \tau_{B}
$$

For $C_{B}>>C_{M}$ and $V_{B}<<V_{M}$ the observed capacitance (Eq. 6) approximates $\approx C_{M}$, the amplitude of the observed capacitance transient becomes:

$$
\Delta C \approx-\frac{\Delta V C_{M}}{C_{B}} \frac{d C_{M}}{d V_{M}}=\frac{\Delta V C_{M}^{4}}{2 C_{B} C_{M 0}^{2} V_{M 0}}
$$

using the proposed Mott-Schottky relation (Eq.3) for the main junction. Therefore in a first approximation we can describe the observed capacitance transient as:

$$
C(t) \approx \frac{\Delta V C_{M}^{4}}{2 C_{B} C_{M 0}^{2} V_{M 0}} \exp \left(-\frac{t}{\tau_{B}}\right)
$$

The time constant depends only directly on the properties of the additional barrier $\tau_{B}=$ $\frac{V_{B} C_{B}}{I}$, while the amplitude is mainly determined by the ratio of the capacitances of the two junctions. Hence, the barrier has stronger influence on the observed spectra than the main junction. Consequently, the fitting algorithm yields smaller uncertainties on its parameters. Since this barrier in the structure is only a perturbation, good estimates for the parameters of the main junction can be obtained from static I-V and C-V characteristics of the whole structure. Therefore, we only need the fitting procedure to gain information on the barriers. It should be noted that this is only typical for the devices studied here and not inherent to the fitting method.

A full calculation in combination with voltage dependent time constants of the interface makes it possible to obtain accurate values for the parameters describing the $\mathrm{I}-\mathrm{V}$ en $\mathrm{C}-\mathrm{V}$ of the additional barrier. An overview of the obtained parameters for the barriers is given in table I. Parity diagrams, showing the good agreement between simulations and experiments, are shown in Fig. 2. To test this method the fit results for the model circuit are compared to the parameters extracted from static measurements on the individual component $\mathrm{B}$. The current voltage characteristics for an 1N5819 Schottky diode were recorded as a function of temperature to determine the barrier height $\Delta E$ and the pre exponential factor $A$.

$$
I_{S}=A T^{2} \exp \left(-\frac{\Delta E}{k_{B} T}\right)
$$


TABLE I: Parameters for the additional non-ohmic barrier in the structure $(95 \%$ confidence intervals are included).

\begin{tabular}{clcccc}
\hline \hline & $\Delta E(\mathrm{meV})$ & $\ln \left[A\left(\mathrm{AK}^{-2}\right)\right]$ & $C_{0 B} \quad(\mathrm{nF})$ & $\alpha \quad(\mathrm{nF} / \mathrm{V})$ \\
\hline Schottky Cr-Si & $638 \pm 2$ & $0.58 \pm 0.01$ & $0.715 \pm 0.148$ & - \\
CdTe CdS & $380 \pm 28$ & $-9.78 \pm 1.64$ & $0.734 \pm 0.380$ & $1.7 \pm 1.4$ \\
CIGS In $\mathrm{S}_{3}$ & $177 \pm 3$ & $-11.1 \pm 0.54$ & $3.52 \pm 1.38$ & $-4.1 \pm 1.8$ \\
CIGS CdS & $19 \pm 4$ & $-20.9 \pm 0.7$ & $19 \pm 10$ & $-210 \pm 172$ \\
\hline \hline
\end{tabular}
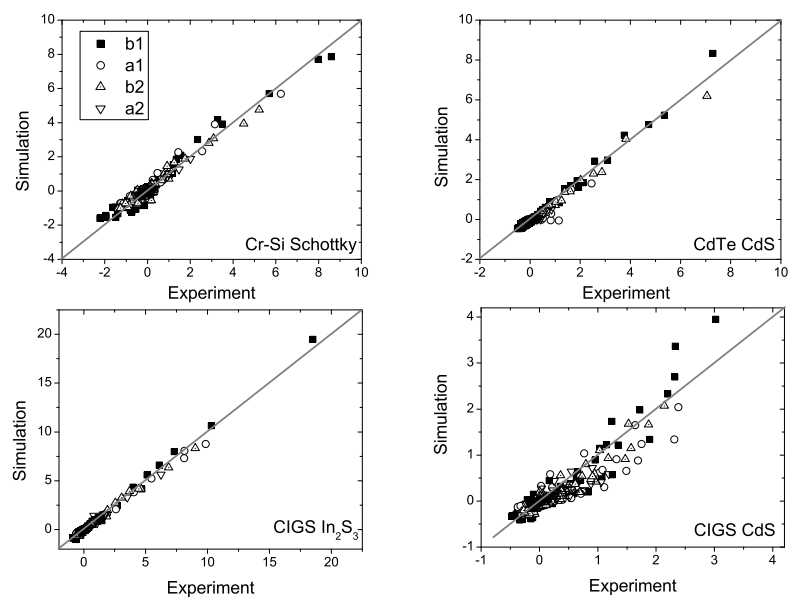

FIG. 2: Parity diagrams for the output parameters $b_{1}, a_{1}, b_{2}$ and $a_{2}$ for the model circuit and the solar cells.

For each temperature, $I_{S}$ was determined as shown in Fig. 3a, and from this temperature dependence $\Delta E$ and $A$ were determined (Fig. 3b). An excellent agreement was found with the predictions based on the parameters using the fitting algorithm. Figure $3 \mathrm{~b}$ shows the Arrhenius diagram for the saturation current determined from the static curve $\left(\Delta E=0.65 \mathrm{eV}, A=1.1 A K^{-2}\right)$ and the one calculated from the parameters received from the fitting algorithm (see table I). For the capacitance of the additional barrier also a good correspondence was found. An average capacitance for this type of component used as additional barrier was $0.75 n F$. Although not all data points could be included in the fitting algorithm, the simulations in Fig.1 confirm the overall good agreement with the experimental DLTS spectra. 

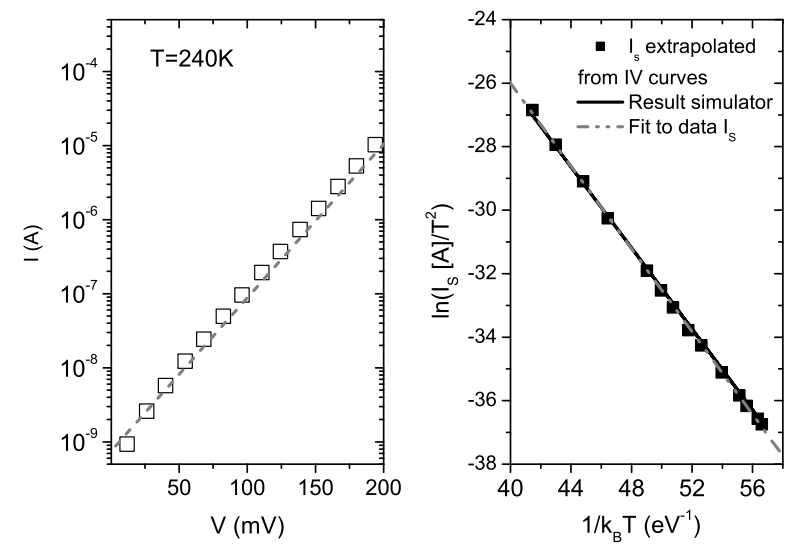

FIG. 3: (a) IV at 240K (b) $I_{S}$ as a function of temperature simulated via the resulted fit parameters versus the one obtained via static measurements

For the $\mathrm{CdTe} / \mathrm{CdS}$ solar cell it is well established that the back contact has an influence on the properties of the solar cell.[20] Recently it has been shown that the N1 signals observed for CIGS solar cells with an $\operatorname{In}_{2} \mathrm{~S}_{3}$ or anCdS buffer layer exhibit the typical properties of an additional barrier.[4] For these three types of solar cells we found a good agreement between the experiments and the model, as can be seen from the parity diagrams (Fig. 2). These good correspondences show that the device modeling using two diodes with I-V characteristics of thermionic emission is valid for the barrier in these thin film structures. We conclude that fitting a model for the circuit to the DLTS spectra with different biases allows to determine parameters for the additional contact responsible for the resonant peak. From this, the barrier height can be accurately determined, without removal of layers and without influencing the total structure. This method makes it possible to characterize barriers within finished products. The possibility to study complete devices can not only contribute to the discussion of the origin of the N1 signal in CIGS and related thin-film solar cells but can also support the engineering of other complicated electronic thin film structures.

The authors acknowledge the special university fund of UGent (BOF-01N01611) for financial support. Antec-Solar is acknowledged for the CdTe/CdS solar cells and A.N. Tiwari, A. Chirilă, F. Pianezzi and S. Buecheler (EMPA Switserland) for kindly providing 
the CIGS solar cells.

[1] D. V. Lang, J. Appl. Phys. 45, 3023 (1974).

[2] P. Blood and J. W. Orton, The Electrical Characterization Of Semiconductors: Majority Carriers And Electron States (Academic, New York,1992).

[3] J. Lauwaert, S. Khelifi, K. Decock, M. Burgelman and H. Vrielinck, J. Appl. Phys. 109, $063721(2011)$

[4] J. Lauwaert, L. Callens, S. Khelifi, K. Decock, M. Burgelman, A. Chirila, F. Pianezzi, S. Buecheler, A. N. Tiwari and H. Vrielinck, . Prog. Photovoltaics: Res. Appl. 20(5) (2012) 588594 .

[5] K. Yamasaki, M. Yoshida, and T. Sugano, Jpn. J. of Appl. Phys. 18(1), 113-122 (1979).

[6] F. Murray, R. Carin, and P. Bogdanski, J. Appl. Phys. 60(10), 3592 (1986).

[7] A. Ricksand and O. Engström, J. Appl. Phys. 70(11), 6915 (1991).

[8] O. Engström, N. Sedghi, I.Z. Mitrovic, and S. Hall, Appl. Phys. Lett. 102, 211604 (2013).

[9] M. Cho, R. Degraeve, P. Roussel, B. Govoreanu, B. Kaczer, M.B. Zahid, E. Simoen, A. Arreghini, M. Jurczak, J. Van Houdt, and G. Groeseneken, Solid-State Electron. 54 1384$1391(2010)$.

[10] R. Rao, R. Simoncini, and F. Irrera, Appl. Phys. Lett. 97, 163502 (2010).

[11] J. Lauwaert, L. Van Puyvelde, J. Lauwaert, J.W. Thybaut, S. Khelifi, M. Burgelman, F. Pianezzi, A.N. Tiwari and. H. Vrielinck,Solar Energy Mater. Sol. Cell. 112 (2013) 78-83.

[12] A. Chirilă, D. Guettler, D. Bremaud, S. Buecheler, R. Verma, S. Seyrling, S. Nishiwaki, S. Haenni, G. Bilger, and A. N. Tiwari, Proc. 34th IEEE Photovoltaic Specialists Conference, Philadelphia, USA, June 712, 2009, 812-816

[13] S. Buecheler, F. Pianezzi, C. Fella, A. Chirilă, K. Decock, M. Burgelman, A.N. Tiwari, Thin Solid Films 51975607563 (2011).

[14] A. Chirilă, S. Buecheler, F. Pianezzi, P. Bloesch, C. Gretener, A. R. Uhl, C. Fella, L. Kranz, J. Perrenoud, S. Seyrling, R. Verma, S. Nishiwaki, Y. E. Romanyuk, G. Bilger and A. N. Tiwari, Highly efficient $\mathrm{Cu}(\mathrm{In}, \mathrm{Ga}) \mathrm{Se}_{2}$ solar cells grown on flexible polymer films, Nature Materials 10 (2011) 857861 .

[15] J. Versluys, P. Clauws, P. Nollet, S. Degrave and M. Burgelman, Thin Solid Films 451, 434 
(2004).

[16] J. Van Gheluwe and P. Clauws, Thin Solid Films 511, 6256 (2007).

[17] S.M. Sze, D.J. Coleman and A. Loya, Solid St. Electron. 14, 1209-1217 (1971).

[18] D.W. Marquardt, Journal of the Society for industrial and applied mathematics 11(2), 431441 (1963)

[19] S. Weiss and R. Kassing, Solid St. Electron. 31,1733 (1988).

[20] P.Nollet, S. DeGraeve, M.Burgelman, Thin Solid Films 361362, 293297 (2000). 